\title{
RECUPERACIÓN DEL PASADO OBRERO E INDUSTRIAL DEL BARRIO DE PATRAIX COMO OFERTA AL TURISMO URBANO DE LA CIUDAD DE VALENCIA
}

\author{
Rafael Temes Cordovez \\ Universitat Politècnica de València
}

\section{RESUMEN}

En este trabajo tratamos de profundiza sobre el valor y la oportunidad que supone hoy, para un turismo cultural y urbano creciente en la ciudad de Valencia, la lectura con perspectiva histórica, de la forma del territorio como anticipación de la ciudad construida. En concreto, los usos industriales que a finales del XIX y principios del XX fueron expulsados de la ciudad intramuros, hoy se sitúan en lugares estratégicos para interpretar la forma urbana actual de muchos barrios. El caso concreto del barrio de Patraix, con un paisaje cultural propio vinculado con la industria urbana y la vivienda obrera, se analiza para establecer dos itinerarios urbanos que fomenten el turismo interesado en el patrimonio industrial.

Palabras clave: Turismo urbano; turismo cultural; patrimonio industrial; SIG; transformaciones urbanas.

\section{Recovery the industrial and worker past of the neighbourhood of Patraix as an offer} to the urban tourism in the city of Valencia

\section{ABSTRACT}

This paper attempts to deepen the value and opportunity of today to a growing cultural and urban tourism in the city of Valencia, reading a historical perspective of the form of the city. In particular, industrial uses that in the late nineteenth and early twentieth centuries were driven out of the walled city, today placed in strategic locations interpreting the current form

Recibido: 20 de marzo de 2015

Devuelto para su revisión: 4 de mayo de 2015

Aceptado: 15 de junio de 2015

Departamento de Urbanismo. Universitat Politècnica de València. Camino de Vera, s/n. 46022 VALENCIA (España).E-mail: rtemesc@urb.uvp.es 
of many urban neighborhoods. The case of the district of Patraix, its own cultural landscape linked to urban industry and workers' housing, is analyzed to establish two urban routes promoting tourism interested in the industrial heritage.

Keywords: Urban tourism; cultural tourism; industrial heritage; GIS; urban transformations.

\section{INTRODUCCIÓN}

Posiblemente Valencia sea una de las ciudades donde mejor se entienda la estrecha relación que ha existido siempre entre los núcleos urbanos y las estructuras previas de asentamiento y transformación del territorio. Como certeramente decía Manuel de Solá a principios de los ochenta, la construcción de la imagen de un lugar pasa por entender e interpretar «... toda la historia social que está escrita en la disposición de los caminos, en los lugares de cruce y de intercambio; en la roturación de cultivos, en la construcción de canales o el regadio de huertas; en las formas de la propiedad, en el emplazamiento de las industrias, el crecimiento de las ciudades y su ocupación del entorno, los contradictorios impactos de las grandes infraestructuras». En definitiva todo lo que existe en el territorio en el que el hombre ha puesto sus huellas, no es fruto de la casualidad o del azar, sino que es el resultado de la adaptación de una u otra actividad al medio. En palabras de A. Corboz (1985): «Los habitantes de un territorio nunca dejan de borrar el viejo libro de los suelos». Es en esta incidencia muta entre naturaleza y hombre, entre formas de vida y territorios, entre paisaje y paisanaje, entre hábitat y hábitos donde reside la definición del término paisaje cultural. La acepción actual de dicho concepto aparece a principios del siglo XX con el profesor Carl Sauer, quien analiza las transformaciones del paisaje natural en cultural debido a la acción del ser humano. En «La morfología del Paisaje» (1925), Sauer define paisaje cultural como el resultado de la acción de un grupo social sobre un paisaje natural. Nos dice que paisaje cultural es el registro del hombre sobre el territorio; un texto que se puede escribir e interpretar; entendiendo el territorio como construcción humana. En el caso que nos ocupa, hablamos de un caso concreto de los paisajes culturales: «los paisajes industriales», que pueden ser de primera generación, los del carbón y el hierro (tayloristas); de segunda generación ligados al petróleo y a la electricidad (fordistas) y los de tercera generación (just in time).

A partir de estos antecedentes, en este trabajo tratamos de profundizar sobre el valor y la oportunidad que supone la lectura, con perspectiva histórica, de la forma del territorio como anticipación de la ciudad construida. Específicamente nos interesa poner de relieve como los territorios, en su día, colindante y periféricos de la ciudad, dictaron normas de ocupación que fueron seguidas por algunos asentamientos. En concreto, los usos industriales que a finales del XIX y principios del XX fueron expulsados de la ciudad intramuros, marcaron coordenadas claves para interpretar la forma urbana actual de muchos barrios.

Hoy estos espacios, ya no periféricos y alejados sino bien comunicados y centrales en la ciudad, coinciden con la revalorización del turismo cultural y urbano que está teniendo una ciudad como Valencia en los últimos años. 
Prueba de ello es que en los últimos cinco años, las pernoctaciones en Valencia han crecido un 19\%, de 3,3 millones en 2009 a más de cuatro millones en 2014, con 1.8 millones de viajeros que han visitado la ciudad durante el mismo año. Los mercados extranjeros han contribuido al total de esta cifra, significando un $60 \%$ de las pernoctaciones ${ }^{1}$.

En esta investigación pretendemos, a través de la visualización de un paisaje cultural propio vinculado con la industria urbana, recuperar varios itinerarios especialmente significativas en el barrio de Patraix de Valencia, que construyan argumentos válidos para poner en valor, «la huella del trabajo en el territorio (ciudad)» (Sabaté, 2011). El valor patrimonial que hoy es reconocido en los edificios que albergaron muchas de las industrias así como las viviendas obrera que hicieron posible la tardía revolución industrial española, se presenta como una oportunidad posible que ofrecer a un turismo urbano interesado en la historia y la evolución de una ciudad como Valencia.

\section{TURISMO URBANO-TURISMO CULTURAL EN VALENCIA}

Actualmente el turismo cultural desarrollado principalmente dentro de los entornos urbanos de muchas ciudades, se ha convertido en un reto para muchos destinos por su atractivo y poder diferenciador. Como queda recogido en el trabajo de Mallor, González y Fayos, (2013) su atractivo económico radica en varios aspectos: primero, a diferencia de otras formas de turismo y a pesar de que estudios recientes matizan esta dimensión del turismo cultural (Cuccia y Rizzo, 2011), existe un consenso alrededor de la idea que el turismo cultural no está tan sometido a la temporalidad o la moda, lo que constituye su principal fuerza (de Cluzeau, 2000; Fernández, Cervantes, Muñiz y Rodríguez, 2006). Segundo, debido a la multiplicación de los centros de interés, el turismo cultural es un mercado extendido por la ciudad que ofrece muchas posibilidades de desarrollo (de Cluzeau, 2000; Greffe, 2002). A pesar de que hoy despierta en algunas ciudades como Valencia un gran interés dada las buenas cifras de visitantes que registra, el turismo cultural no es nada nuevo. Podemos considerar al turismo cultural en su escala urbana, como el heredero del Grand Tour, los viajes que realizaban los jóvenes aristócratas ingleses por la Europa continental para terminar su formación desde mediados del siglo XVII hasta el siglo XIX. Al Grand Tour le debemos el interés por el patrimonio arquitectónico, a la vez que la evolución y el desarrollo de la cartografía urbana (Pié, Vilanova y Díaz, 2010). Esta preocupación por el patrimonio se hace presente sobre todo cuando se moderniza la ciudad con grandes actuaciones en el tejido urbano existente y se alzan las primeras voces a favor de la conservación del patrimonio. La cartografía urbana, antes de su utilización como documento técnico para la urbanización moderna, fue una guía turística para el visitante. La planta de Bufalini de 1551 y el mapa de Roma de Nolli de 1748 (Figura 1) son dos ejemplos del valor del mismo, como guía para el visitante, plano en el que se subraya la relación entre lo nuevo y lo antiguo, entre la ciudad real y los restos arqueológicos o el espacio de las instituciones monumentales.

1 Datos extraídos de las Estadísticas de Turismo 2014 (Fundación Turismo Valencia). 
Figura 1

FRAGMENTO DEL MAPA DE ROMA DE NOLLI, 1748

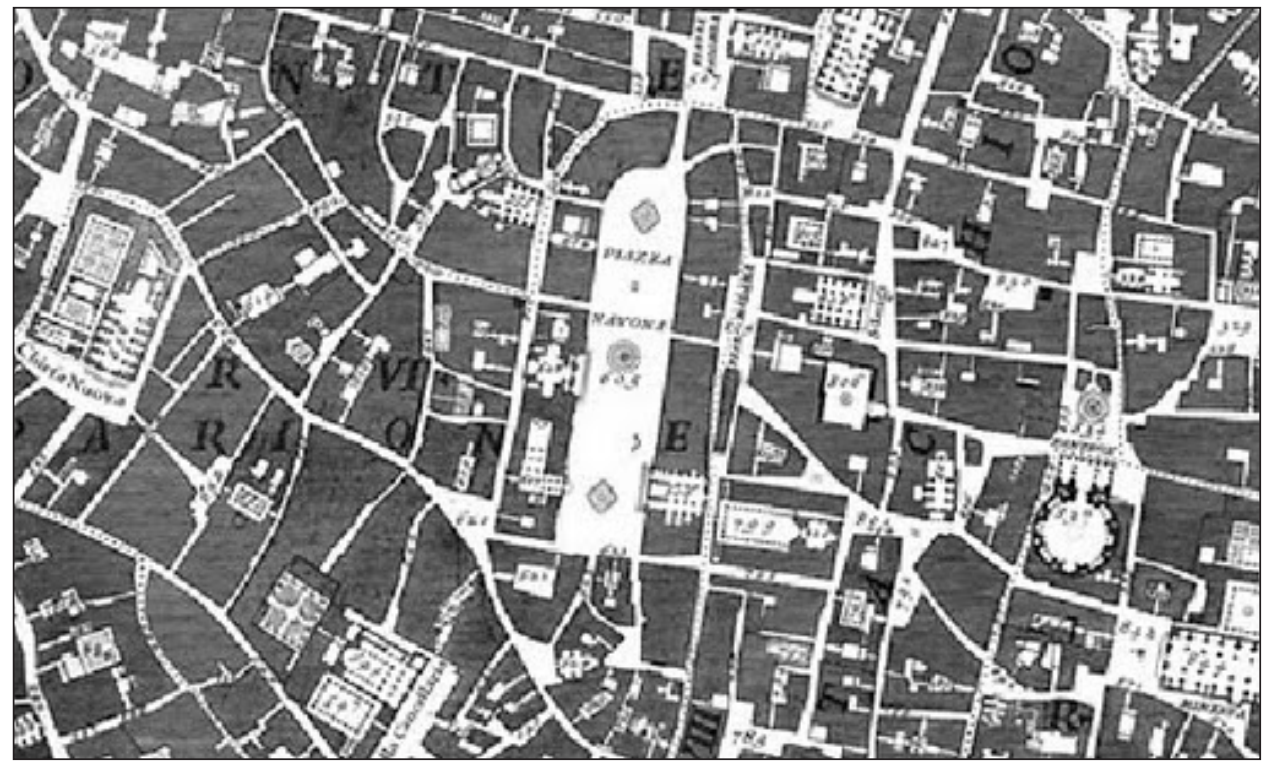

Fuente: Vilanova y Díaz, 2010.

No existe un consenso claro entorno a la definición de turismo cultural, en cuanto a que depende, en buena parte, de las necesidades y motivos propios de los consumidores. El grado de subjetividad por tanto es amplio al respecto. En esta misma línea tampoco ha existido en los últimos años una relación de tipologías de turismo cultural ampliamente aceptada (McKercher y Cros, 2002) por lo que la taxonomía del turista cultural es un tema continuamente revisitado (Stylianou-Lambert, 2010). En los trabajos de Mallor, González y Fayos (2013), se ofrece una tabla comparativa en la que se recogen las dimensiones principales del turismo cultural según varios autores, pudiendo llegar a la conclusión del predominio de los aspectos clásicos del turismo cultural como la arquitectura, la artesanía y los museos. De esta triada habitualmente los museos y la arquitectura, y por extensión el urbanismo, tienen su escenario principal de referencia en las ciudades, por lo que a menudo podemos utilizar de forma equivalente, turismo cultural y turismo urbano. En los últimos años también en los museos se ha producido un cierto cambio en la manera de hacer accesible su patrimonio. La búsqueda de una mayor naturalidad y un mayor impacto ha llevado a importantes museos como el Museum of London o el Urban Museum McCord de Montreal a generar nuevas «experiencias» en las que el museo se vive en la propia calle, aprovechando el tránsito urbano como hilo conductor para mostrar la cultura de una sociedad ${ }^{2}$.

2 Ver: Streetmuseum (http://www.museumoflondon.org.uk/Resources/app/you-are-here-app/home.html) MLT Urban museum (http://www.mccord-museum.qc.ca/en/mobile/MTLUrbanMuseum/) 


\section{PATRIMONIO INDUSTRIAL Y EL TURISMO INDUSTRIAL}

El patrimonio es un concepto muy amplio que tradicionalmente ha estado muy atento a los valores que se podrían denominar «histórico-artísticos». Sin embargo, no es nueva la reinvindicación de la no exclusión de lo patrimonial a lo producido por la industria en los dos últimos siglos (Casanelles Rahola, 1988, p. 13; Aguilar Civera, 2004). Como definición de «patrimonio industrial» podemos tomar aquella de la carta de Nizhny Tagil aprobado por los delegados de la asamblea de TICCIH en Moscú en el año 2003 que en buena medida es la asumida por el Plan Nacional de Patrimonio Industrial (2002): «... todos los restos materiales, bienes muebles e inmuebles, con independencia de su estado de conservación, formas o elementos de la cultura material de la sociedad industrial capitalista, generados en el desarrollo histórico por las actividades productivas y extractivas del hombre, así como aquellos testimonios relativos a su influencia en la sociedad».

A partir de aquí, el turismo industrial es aquel interesado en conocer dicho pasado fabril, sus cambios y su influencia en la cultura. Como afirma Pardo Abad (2004) el turismo industrial se ha organizado en bastantes casos mediante rutas o itinerarios industriales. Dichas rutas se pueden categorizar en: rutas urbana, comarcales, regionales y temáticas. En concreto, las rutas urbanas ofrecen la posibilidad de conocer el escenario por excelencia de la Revolución Industrial: la ciudad. En los tejidos urbanos se mezcla la incorporación de la industria y la residencia obrera necesaria para dotarla de mano de obra. Así mismo la vinculación con los medios de producción, sobre todo el agua, a través de acequias o pequeñas rieras, dota de personalidad propia a algunas tramas urbanas que cobran relevancia una vez se descrifra el origen de su forma urbis. Algunas ciudades españolas como Barcelona, Bilbao o Avilés, con un potente y reconocido pasado industrial han trabajado en la línea de fomentar las rutas turísticas vinculadas a lo industrial como complemento a otros cauces de entrada de visitantes en la ciudad. En el caso de Valencia, siendo su pasado industrial también muy relevante (Aguilar Civera, 2004), dicha vertiente del turismo no ha tenido aún su desarrollo.

A continuación describimos las bases que podrían justificar una propuesta para promocionar parte de dicho legado fabril desarrollado en el barrio de Patrix de la ciudad de Valencia.

\section{PATRAIX}

\subsection{La huerta de patraix, sus caminos y acequias}

Patraix igual que otros tantos barrios de Valencia fue desde la Edad Media hasta finales del XIX uno de los pequeños núcleos de población de la Huerta que, como satélites estratégicamente situados, rodeaban al núcleo central. Apoyados en estos asentamientos, se cultivaba y explotaba la Huerta construyendo un paisaje singular. Como bien describe Eric Guinot, (2007) «su peculiaridad fundamental reside en la compleja interrelación entre ordenación del territorio en base a la red hidráulica organizada y jerarquizada que las vertebra, con el establecimiento de la red de poblamiento, las vías de circulación y 
la estructura de los parcelarios de tierras de cultivo». Ahora bien, lejos de iniciar una descripción compleja y exhaustiva sobre los orígenes y formación de la Huerta, hecha por otro lado, con gran acierto por otros autores con anterioridad, nuestro punto de partida es la del territorio de la Huerta en proceso de urbanización a finales del siglo XIX. En ese momento, para entender qué es lo que sucedía en los alrededores de Patraix, hemos de comprender qué ocurría en la Valencia central (Figura 2). La relación de equilibrios y conveniencias que siempre ha mantenido la ciudad capital con las poblaciones satélite, implica una estrecha vinculación entre estos espacios. Lo que en un territorio era necesario se producía en el otro, y lo que no cabía en la ciudad central lo acogía el espacio de la Huerta próxima.

En este sentido, Patraix debe comprenderse no sólo como un pequeño asentamiento de la Huerta, sino como una porción de territorio rural, dominado fundamentalmente por la acequia de Favara, junto a otras acequias, como la de Mislata, Andarella y Faitanar, que se componía de un núcleo de casas agrupadas en torno a una plaza, unas pocas calles y un rosario de alquerías en las aproximadamente 975 hectáreas que lo formaban (Algarra Pardo, 2003).

\section{Figura 2}

\section{FRAGMENTO DEL PLANO DE VALENCIA Y SUS ALREDEDORES. CUERPO DE ESTADO MAYOR DEL EJÉRCITO, 1883}

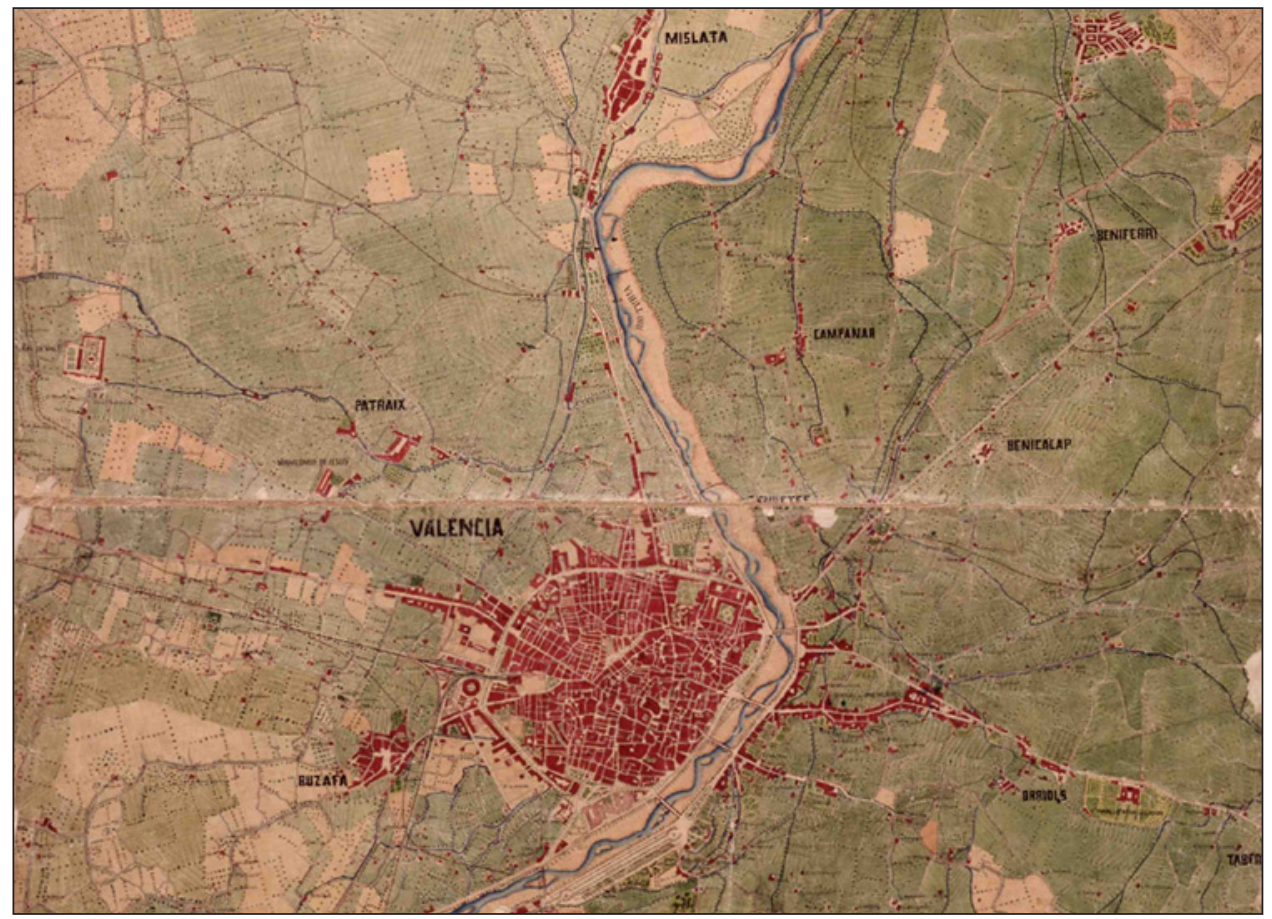


Junto a la estructura constituida por las acequias y sus brazos, a Patraix se accedía desde Valencia por cuatro caminos hoy transformados en calles. En dirección este a oeste el primero era el Camino de Picassent que partía del Camino Real de Xátiva o de Madrid (calle de San Vicente Mártir) a la altura de la calle Marvá y al atravesar el núcleo de Jesús, enlazaba con el camino (hoy calle del Beato Nicolás Factor) que conducía al extremo este de Patraix. Por su parte estaba el Camino de Jesús que desde la ronda de las antiguas murallas de Valencia finalizaba en el Convento de Jesús. Saliendo desde el antiguo Hospital General estaba el Camino del Cementerio del Hospital ·actual calle de Cuenca), también conocido como Camino de Patraix. Por otro lado el Camino de Torrent que partía desde la calle Ángel Guimerá, junto a la ronda de las murallas, y discurría por el lado oeste de I>Hort de Pontons y de Patraix, constituyendo la vía principal de acceso a las huertas de los pobladores de Patraix junto al Assagador de les Monjes (Algarra Pardo, 2003).

\subsection{La configuración obrero-industrial de Patraix}

Lo que podemos entender como la estructura básica de Patraix formada por las pequeñas manzanas de alrededor de la plaza y las infraestructuras de acequias y camino que lo conectaban y relacionaban con el resto de los territorios, realmente permaneció prácticamente intacta hasta finales del siglo XIX. Las razones que argumentan la nueva pauta de crecimiento que se va a desarrollar a partir de principios del XX, se apoya en la expulsión de la industria de la ciudad central y el aumento de población demandante de empleo para la misma.

La introducción de nuevas tecnologías como el uso de la máquina de vapor y sus distintas aplicaciones en Valencia, supuso un importante adelanto que incidió en algunos aspectos de la configuración urbana existente. La máquina de vapor, proporcionará avances significativo en las explotaciones agrícolas e industriales. Las transformaciones del paisaje próximo a Valencia generadas como consecuencia del empleo de la bomba de vapor para la extracción de aguas, quedan expuestas de manera clara en los trabajos de Enrique Giménez (1996). En la ciudad intramuros el paisaje también va a cambiar: nuevos tamaños de edificios que albergan talleres, chimeneas de ladrillo, columnas de humo... Los motores se incorporan a la fabricación de hilaturas, torcido de seda, fundición de hierro, azulejos, etc., y un sin fin de pequeñas industrias dedicadas a actividades diversas, como la confección de velas, el curtido de pieles o el montaje de abanicos. Pero su proliferación, no va acompañada inicialmente de una ordenación consecuente con los problemas y los riesgos que generaban el uso de dicha tecnología en convivencia con los tejidos urbanos. La precariedad de la situación se evidencia y la respuesta a la misma se traduce en tres acciones vinculadas: generación de las primera propuesta de ensanche, expulsión de las industrias fuera del recinto intramuros y nacimiento de las primeras iniciativas de fomento de la vivienda obrera.

Respecto a la nueva propuesta de ciudad, los ensanches junto con las reformas interiores, tratan de superar las estrecheces y los problemas de salubridad e higiene que se denunciaba en el centro urbano, especialmente agravados por la incorporación de la industria y el hacinamiento de la población obrera. Ahora bien, sabemos que 
las dos propuesta de ensanche que tuvo Valencia, la primera en 1884 y la siguiente en 1907, no van a ser suficientes para resolver este problema. En el primer ensanche, como refleja Rafael Temes (2007), un tercio de la capacidad potencial del proyecto ya estaba ocupada. Por su parte el segundo ensanche, más allá de la Gran Vía Fernando el Católico, contaba con una situación de ocupación previa considerable y se había complicado mucho los trámites para edificar, por lo que se retrasará su construcción. A estas razones se le añadirá la permisividad municipal para construir barriadas en las afueras del ensanche, por lo que el salto hacia el exterior de la ciudad planificada propiciará operaciones más cómodas, rápidas y rentables. Por su parte, la industria que no podía mantenerse en el recinto urbano, no encuentra tampoco en los ensanches oportunidades para su ubicación. Serán los suelo más allá de Tránsitos los espacios elegidos. Aquí descubrimos una configuración singular en el espacio urbano-rural de Patraix, donde conviven usos agrícolas, industriales, residenciales y ganaderos. Entre los años 20 y 40 del siglo XX, en esta zona se configurará un paisaje urbano hoy difícil de imaginar que consolidan algunas de las señas de identidad del actual barrio. Pasemos a detallar algunos de estos escenarios

\subsection{Los usos industriales en Patraix a principios del siglo $\mathrm{XX}$}

El establecimiento y disposición de usos industriales en la zona se desarrolla de forma polarizada entorno a los principales caminos de unión de la Huerta y la ciudad de Valencia. Siguiendo esta lectura y guiados por la restitución hecha al efecto de la cartografía catastral del Valencia levantada por el entonces Instituto Geográfico Catastral en 1929-44 (Temes Cordovez, 2008) tenemos la siguiente descripción:

En torno a la Carretera de Madrid a Valencia por Casas del Campillo, justo pasado el trazado de Tránsitos (Pérez Galdós) podemos localizar: varios almacenes y molinos arroceros; un Fábrica de Licores; la famosa fábrica de «Tintas Sama»; varios Talleres de Fundición y una Fábrica de Muebles curvados que competía con la también famosa Fábrica de Ventura Feliu situada en la misma carretera a unos 900 m. más al sur. (Figura 3). En torno al Camino Viejo de Pisassent, justo en su encuentro con Tránsitos (Pérez Galdós) se situarán la famosa Fábrica de Chapas de S. Vilarrasa generadora del aglomerado de 3 capas NOVOPAN; una Fábrica de pañuelos y un grupo de Fábricas de muebles entre las que destacan la de E. Vilella y de Remigio Sapiña. Más adelante, pasado la Iglesia de Santa María de Jesús, nos encontramos con un Almacén de maderas con una superficie considerable y una Fábrica de Chapas y Prensas. Cerrando esta sucesión, y dentro del ámbito del análisis situaríamos a la Fábrica Térmica «La Valenciana».

Alrededor del Camino Viejo del Cementerio (actual calle Cuenca) sobrepasada la vía del Ferrocarril Valencia-Utiel (calle Tres Forques), se habían asentado desde finales del siglo XIX diversas industrias, como la de elaboración de sacos de yute de la Viuda de Alcañiz, o la Fábrica de curtidos de José Barrera, construida en el año 1888. Asimismo, en la calle Cuenca se ubicó una Granja Avícola, que posteriormente se reedificó para Casacuartel de la Guardia Civil. Más adelante, el camino se junta con el trazado del acequia de Favara al encontrarse con la Fábrica de muebles de Mariano García y otra de juguetes de Alberto García a la que le sigue la Batifora, la primera fábrica de seda y algodones 


\section{Figura 3 \\ FÁBRICA DE MUEBLES CURVADOS DE VENTURA FELIU. SECCIÓN DE CURVADOS, PRINCIPIOS DEL XX}

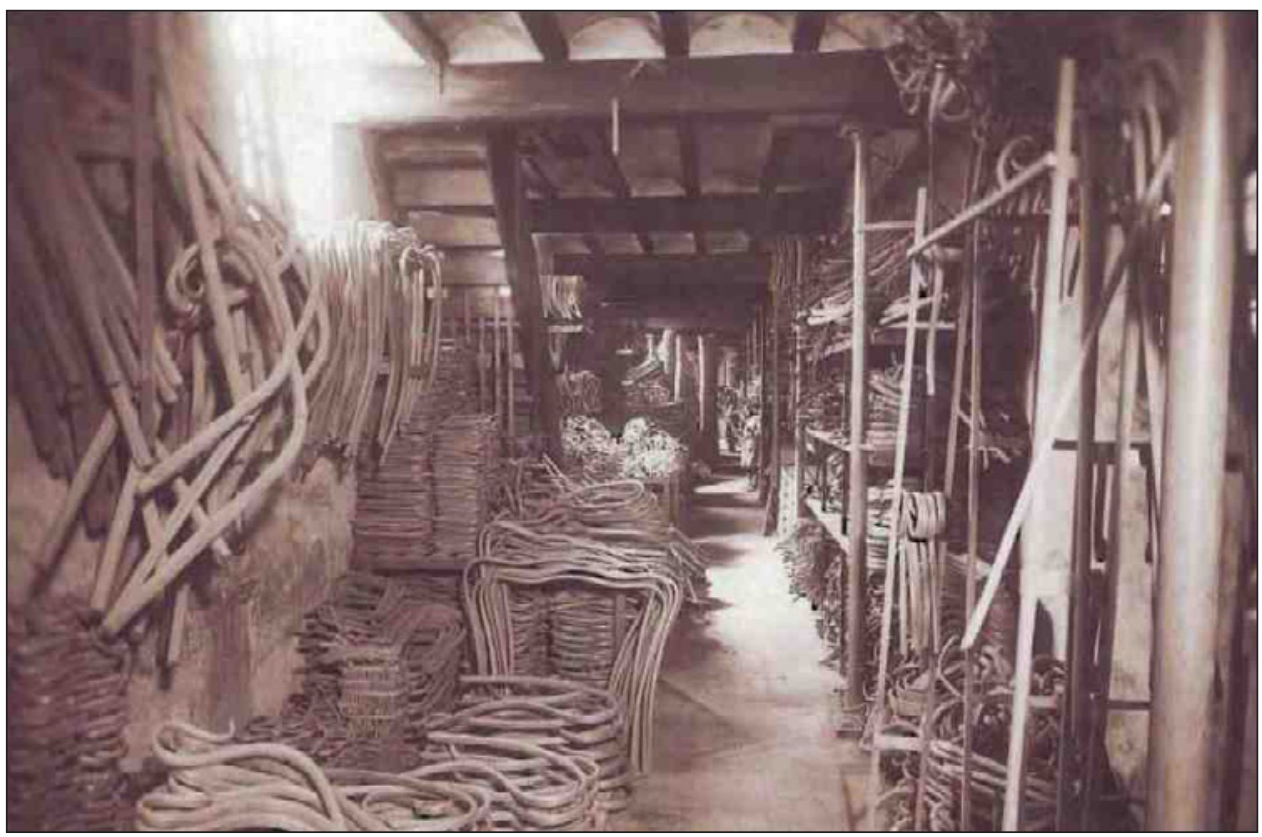

con una caldera de vapor capaz de producir fuerza con la que mover todos sus telares y devanadoras. Esta fábrica usaba el agua de la acequia de Favara, que una caldera alimentada por leña y carbón se encargaba de caldear para generar vapor y al mismo tiempo se podían hervir los capullos del gusano de seda. La industria, que llegó a ser muy importante en su momento, tuvo hasta trescientos empleados, en su mayoría mujeres procedentes de Picanya y Paiporta, más unas cincuenta, especializadas en la cría del gusano de seda, que procedían de Segorbe. Ya al final de este camino y antes de cerrar el ámbito de análisis, nos encontramos con el Horno de Vidrio Alsina y la Granja de Vacas de la Diputación Provincial.

Finalmente en torno a la Acequia de Favara, Camino de Mislata y Viejo de Torrente se concentraban otras industrias. Justo en el cruce con Tránsitos nos encontramos con la Fábrica de muebles de Estornell; varios almacenes de madera y una Fábrica de trenzado de yute.

En la Figura 4 se puede apreciar bien la disposición de estas instalaciones industriales y valorar su tamaño respecto a los asentamientos tradiciones que existían en el momento. $\mathrm{Si}$ a esto se le une la percepción de chimeneas y cubiertas continuas que caracterizaban buena parte de dichas industrias, se puede llegar a entender la fuerte presencia que en el paisaje urbano tuvieron las industrias en este momento. 


\section{4 . Los asentamientos obreros en Patraix a principios del siglo XX}

Junto a estas industrias e instalaciones se fueron construyendo, en las primeras décadas del siglo pasado, conjuntos de casas adosadas, en ocasiones denominadas barriadas, que evitando las restricciones de la zona de los ensanches encontraban grandes ventajas en su construcción más allá de Tránsitos. Mientras esto ocurría, a principios del siglo XX, la ciudad seguía acogiendo crecimientos. La demanda de trabajadores para las industrias y la mejora de las infraestructuras de acceso se traducen en un incremento continuado de la población sólo alterada por las epidemias y los efectos de las guerras. La población obrera expulsada en parte del interior de la ciudad mediante las reformas, y la nueva población flotante que se va destilando de los municipios próximos, generan un frente de habitantes que necesita alojamiento. En la periferia se sitúa un nuevo escenario de acogida. Los costos menores del suelo y la posibilidad de introducir nuevas morfologías en consonancia con la ideología reformista de finales de siglo, inauguran en estos espacios una etapa de nueva colonización que condicionarán en parte los crecimientos futuros. Proliferan las sociedades constructoras, cargadas por lo general de un fuerte ideario político social, en ocasiones de tipo benéfico y otras veces moralizante, paternalista o de adoctrinamiento burgués, pero en cualquier caso dedicadas a la edificación de casas para obreros. Apoyadas en las sucesivas leyes de Casas Baratas y Económicas, se establecen mecanismos para potenciar la construcción de alojamiento obrero a partir de la iniciativa privada. El barrio de Patraix, donde se concentra buena parte de la industria de la ciudad, va a ser un lugar elegido para la instalación de algunas iniciativas de Casas Baratas. Siguiendo la misma lectura que hicimos para la industria podemos ver sobre la cartografía de Valencia 1929-44 varias cosas (Figura 4):

En torno a la Carretera de Madrid a Valencia por Casas del Campillo, nos encontramos con la Cooperativa de Casas Baratas «San Fernando», formada por diez unidades construidas en 1928 y formada por una tipología muy simple de viviendas de una planta con patio trasero. Trescientos metros más al sur, y construido un año antes se encuentra las Casas Baratas «La Previsora». En este caso, como comenta Juan Blat (2000), se trata de una propuesta para clases medias donde se percibe el encanto de los modelos de ciudad jardín con el uso de pareados con jardín y ventanas bay-windows, desarrollados por Unwin \& Parker pocos años antes en la periferia de Londres.

A escasos cuatrocientos metros y situado entre el Camino Viejo de Picassent y las vías del Ferrocarril a Villanueva de Castellón, se situó en 1926 el grupo de Casas Baratas «La Emancipación» construidas por el arquitecto Emilio Artal siguiendo también los modelos británicos de casas pareadas (Figura 5).

Más al norte, próximo al casco urbano de Patraix y entre el Camino de Mislata y el Camino Viejo de Torrente se sitúan tres nuevas agrupaciones dando forma a una de las concentraciones más interesantes de esta naturaleza en Valencia. Por una parte tenemos el Grupo «Ramón de Castro», construido en 1906 y formado por 34 viviendas entre medianeras que le acompaña, al otro lado de las vías del tren, el Grupo de «Villas Pontón» formado por unas 40 viviendas. Siguiendo el trazado de la acequia de Favara y limitado por el Camino de Mislata nos encontramos más al norte con las 60 viviendas de la «Cooperativa de Dependientes del Comercio» construidas nuevamente por Emilio Artal y manteniendo los modelos de inspiración británica empleado en casos anteriores. 
Figura 4

DISTRIBUCIÓN DE INDUSTRIAS Y GRUPOS DE CASAS

BARATAS EN TORNO A LOS CAMINOS PRINCIPALES, CARTOGRAFÍA CATASTRAL 1929-44

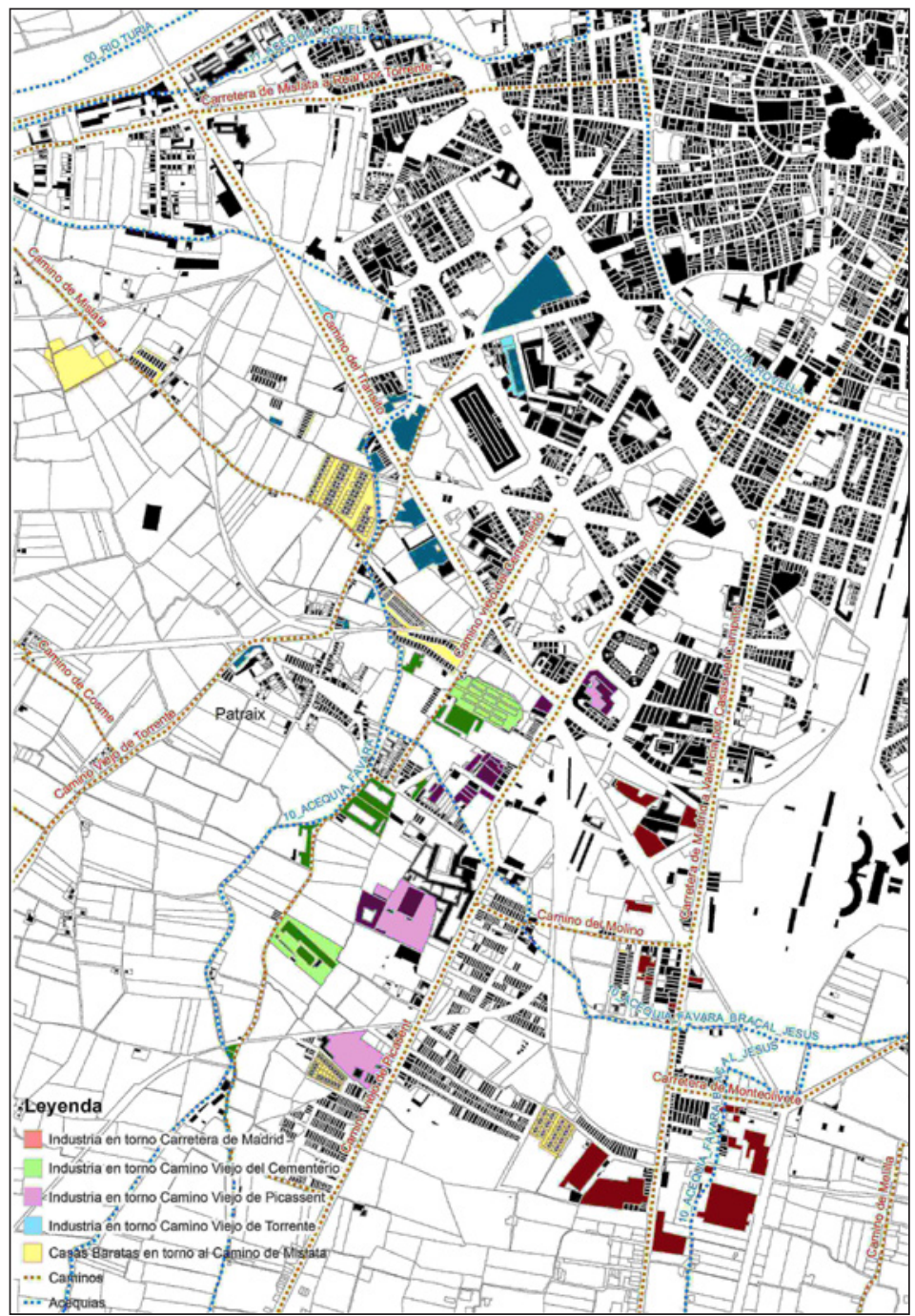

Por último, y siguiendo el Camino de Mislata nos encontramos con tres grupos de Casas Baratas construidas en 1926 en el caso de las de «Julián Peña», en 1928 las del «Arte Mayor de la Seda», y en 1925 las de «Obreras Previsoras» siendo los dos primeros grupos de viviendas de una planta entre medianeras y las segundas de dos plantas entre medianeras. 


\section{Figura 5 \\ GRUPO DE LA COOPERATIVA DE CASAS BARATAS «LA EMANCIPACIÓN», J. BLAT}

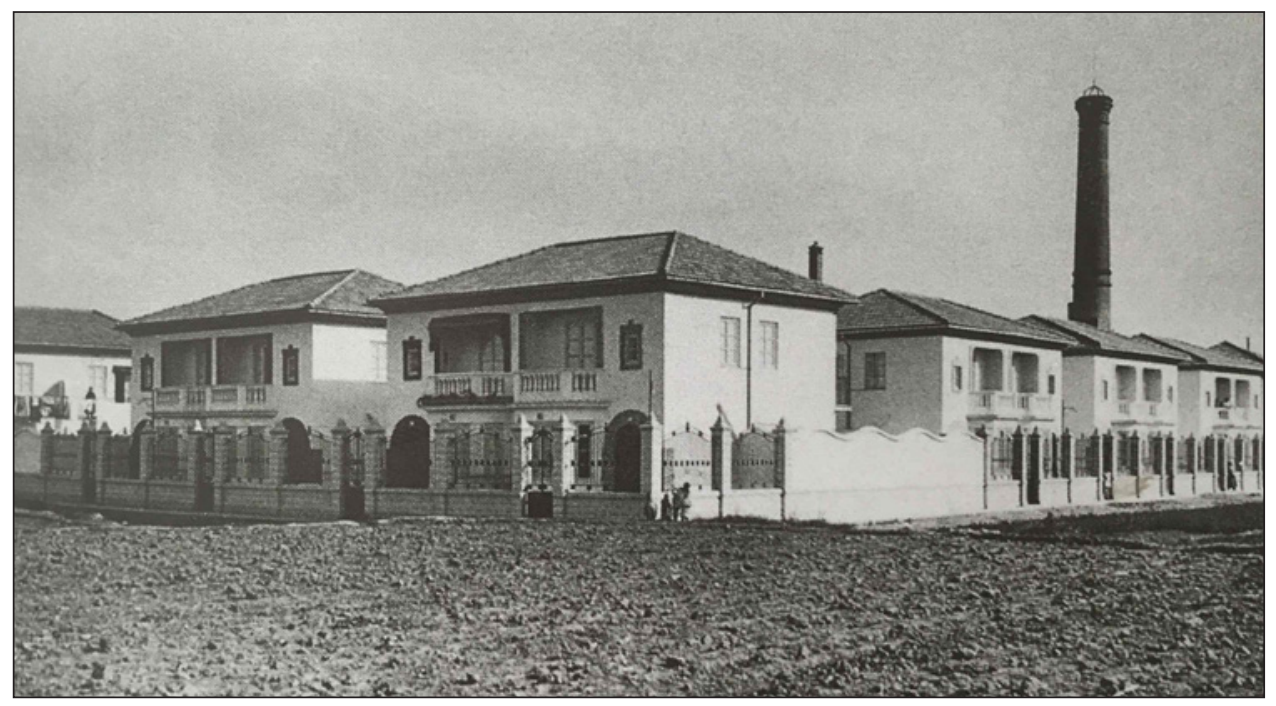

\section{PROPUESTA DE RECUPERACIÓN DE ITINERARIO INTERPRETATIVO DEL BARRIO}

Como señala Margalida Castells (2001) el concepto «Interpretación» fue introducido en España, en el último tercio del siglo XX, de la mano de los responsables del patrimonio natural y bajo la forma de «interpretación ambiental», recogiendo los postulados teóricos que acompañaron, a finales del siglo XIX, la declaración de los primeros parques naturales estadounidenses. Un momento de inflexión lo marcó la obra de Freeman Tilden «Interpreting our Heritage» (1957), considerada todavía hoy en día un excepcional trabajo sobre la teoría de la interpretación. En los años 80, cuándo el patrimonio adoptó una perspectiva integral, la interpretación se consolidó en el panorama mundial, especialmente a partir del Primer Congreso Mundial del Patrimonio que se celebró a Banff (Canadá) en el año 1985. Ya en la Declaración de Brujas (1995) se establecieron varios niveles de protección y uso de los bienes patrimoniales a conservar, con una perspectiva a medio y largo plazo en lo que acontece a su rentabilidad cultural, social y económica. Según la Heritage International Interpretation, se entiende por «Interpretación» el arte de explicar el significado y el sentido de un lugar que se puede visitar, es decir, un método de presentación, comunicación y explotación coherente del patrimonio.

En esta línea de trabajo en la que se plantea el patrimonio cultural como un recurso vinculado estrechamente con el territorio o la ciudad, «más allá del catálogo y el museo» en palabras de Pere Vall (1999), nos planteamos el diseño de unas rutas patrimoniales que interpreten el pasado histórico-industrial de una parte de la ciudad. El trazado de este tipo 
de itinerarios comparte algunos rasgos con el diseño, desarrollo y gestión de lo que se ha dado en llamar «parques patrimoniales». Como asegura Joaquím Sabaté (2004) tras el análisis de más de una centena de casos americanos y europeos, los parque patrimoniales deben tener la aspiración de promover no solo la preservación del patrimonio, la promoción de la educación y actividades recreativas, sino también procurar un nuevo desarrollo económico. «La gestión inteligente del patrimonio está suponiendo en diversos lugares uno de los factores claves para su desarrollo económico, porque atrae turismo e inversiones, porque genera actividades y lugares de trabajo y, fundamentalmente, porque refuerza la autoestima de la comunidad» (Sabaté y Lista, 2001).

Con estos antecedentes consideramos útil hacer nuestras algunas de las conclusiones de diseño y gestión extraídas del análisis anterior como pautas de referencia para nuestras rutas patrimoniales. Entre dichas pautas destacaríamos las fijadas en el Cuadro 1:

\section{Cuadro 1 \\ IDEAS BÁSICAS PARA EL DISEÑO DE UN PARQUE PATRIMONIAL/RUTA PATRIMONIAL}

\begin{tabular}{l|l}
\hline Ideas & Resumen \\
\hline Primera idea & $\begin{array}{l}\text { En toda interpretación patrimonial resulta imprescindible explicar bien una his- } \\
\text { toria. Dicha historia es fundamental para relacionar entre sí recursos alejados, } \\
\text { con el objeto de que interactúen y se refuercen, para situar en cada momento al } \\
\text { turista, al estudioso, al usuario... respecto a un guión general }\end{array}$ \\
\hline \multirow{5}{*}{ Segunda idea } & $\begin{array}{l}\text { La historia que se narra ha de ser original, coherente con los recursos de que se } \\
\text { dispone y, sobre todo, estar muy bien documentada. En este sentido, cabe desta- } \\
\text { car que los propios residentes constituyen recursos culturales muy importantes. } \\
\text { Es necesario recalcar que los recuerdos constituyen recursos culturales básicos; } \\
\text { de ahí la importancia de la labor de recopilación de antropólogos, sociólogos, } \\
\text { historiadores geógrafos y documentalistas }\end{array}$ \\
\hline $\begin{array}{l}\text { Resulta crucial definir una clara estructura física del parque patrimonial/ruta } \\
\text { patrimonial. El conjunto de propuestas analizadas presenta un sistema de or- } \\
\text { ganización, una estructura, con notables similitudes. En la práctica totalidad } \\
\text { de los casos es posible reconocer la existencia de unos mismos componentes } \\
\text { que, forzando algo la interpretación, podríamos hacer equiparables a los cinco } \\
\text { elementos constitutivos de la sintaxis propuesta por Kevin Lynch en su libro La } \\
\text { imagen de la ciudad: } \\
\bullet \text { El ámbito global y los subámbitos del parque: áreas (regions). } \\
\text { • Sus recursos patrimoniales y servicios: hitos (landmarks). } \\
\bullet \text { Las puertas y accesos, los centros de interpretación y museos: nodos (nodes). } \\
\bullet \text { Los caminos que vinculan todo lo anterior: itinerarios (paths). } \\
\bullet \text { Los límites visuales (y administrativos) de la intervención: bordes (edges). }\end{array}$ \\
\hline $\begin{array}{l}\text { La mayor parte de las iniciativas exitosas se caracterizan por } \\
\text { surgir de la base, de los agentes locales, de los denominados grassroots, de } \\
\text { los amantes de su territorio que pretenden valorizar sus recursos. Las mejores } \\
\text { iniciativas se caracterizan por crecer desde abajo hacia arriba. }\end{array}$ \\
\hline Fuarta idea
\end{tabular}

Fuente: Sabaté (2004). 


\subsection{Itinerario obrero industrial del barrio de Patraix (Valencia)}

En base a las 4 ideas resumidas en el Cuadro 1 y tras la descripción hecha en relación a las principales condiciones patrimoniales del barrio de Patraix, hemos diseñado dos itinerarios o rutas urbanas para poner en valor el pasado obrero industrial del barrio. La idea es poder con dichos itinerarios, ofrecer una visión integral de su historia a partir de la visita a las principales fábricas y grupos de Casas Baratas de alojamiento obrero. A partir de estas ideas la propuesta que se ofrece es la siguiente (Figura 6)

\section{Figura 6}

\section{SUPERPOSICIÓN SOBRE ORTOFOTO ACTUAL DE LA POSICIÓN DE LAS FÁBRICAS Y CASAS BARATAS. DISPOSICIÓN DE LOS 2 ITINERARIOS PROPUESTOS}

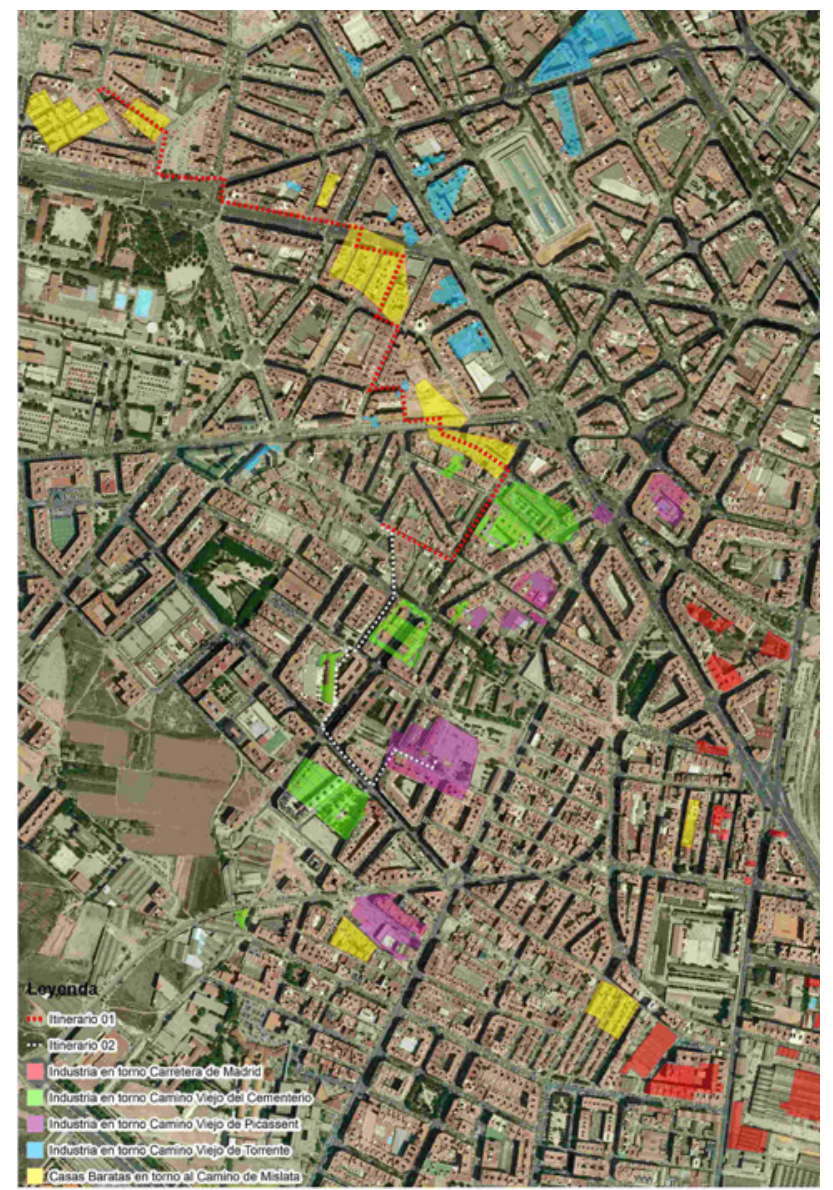

Itinerario 01. El alojamiento obrero a principios del siglo XX en Valencia. Las Casas Baratas de Patraix Itinerario 02. La concentración industrial más allá de Tránsitos. El pasado fabril del barrio de Patraix 
Siguiendo el patrón de ideas resumidas en el Cuadro 1, y analizando en paralelo los dos itinerarios propuestos tenemos:

Respecto a la historia explicada, el primer itinerario es el de la expulsión de la vivienda obrera fuera de los ensanches y su ubicación en los entonces suelos periféricos donde los costos de implantación eran menores. Así mismo, el surgimiento de las primeras políticas de vivienda obrera a través de las leyes de Casas Baratas, mostrándose algunas de las más singulares tipologías de este tipo de vivienda construidas en Valencia.

Por su parte, en el segundo itinerario, la historia también se detiene en el desplazamiento del centro a la periferia, experimentado por la primera industria fruto de la revolución industrial tardía en Valencia. Su disposición entorno a los caminos de acceso y a las acequias como fuente de suministro de agua, así como la descripción de sus usos y arquitectura completan la narración que proponemos.

Respecto a la documentación de dicha historia, afortunadamente en la ciudad de Valencia se cuenta hoy con suficientes trabajos de investigación que permiten reconstruir y documentar con precisión tanto el origen y el pasado de las viviendas obreras (Blat, 2000; Giménez,1996; Temes, 2007) como de las primeras industrias establecidas en la ciudad (Aguilar, 1998; López, 2013)

Respecto a la estructura de la ruta, se organizan las rutas atendiendo a las recomendaciones establecidas. Por un lado, la zona de actuación está dividida en dos «áreas» (la abarcada predominantemente por vivienda obrera y la compuesta por antiguas industrias). Los «hitos» que jalonan los itinerarios están formados por las fábricas, chimeneas así como algunos elementos que dan testimonio de la pasada existencia de espacios fabriles en la zona. Como «puertas de acceso» hemos decidido utilizar la plaza principal de Patraix, núcleo fundacional de la población como el elemento de espacio público de referencia e identificación de la propuesta. Respecto a los itinerarios tenemos el primero que trata de ofrecer una visión amplia de la secuencia de grupos de Casas Baratas implantadas en el barrio de Patraix durante el primer tercio del siglo XX. El itinerario se desarrolla en dirección norte siguiendo el antiguo Camino de Mislata, desde la plaza principal del barrio hasta la avenida del Cid, para visitar seis grupo de viviendas de los que aún es posible reconocer, de manera integral, dos de ellos, otros dos sólo con algunos vestigios y dos sólo su ubicación. Respecto al segundo itinerario utiliza principalmente el Camino Viejo del cementerio y la acequia de Favara en su dirección sur-oeste como eje principal del recorrido. En este caso, el recorrido es capaz de reconocer la localización de seis industrias, entre las que destaca la «Granja Experimental» y la «Batifora», terminado el recorrido en la antigua chimenea de la «Serrería de Franco Tormo» que hoy da nombre a la calle.

Respecto a los límites visuales que contextualizan el ámbito de interpretación en nuestro caso queda delimitado al noreste por la Avd. de Pérez Galdós, uno de los tramos del antiguo camino de Tránsitos que marca un claro borde urbano con el resto de la trama urbana de la ciudad.

Respecto a la participación vecinal, en el barrio de Patraix se cuenta con una asociación denominada «Asociación de vecinos, cultural y de consumidores de Patraix» muy activa y sensibilizada con la recuperación del patrimonio de la antigua población por lo que se cuenta con las bases para poder integrar el proyecto estableciendo el respaldo ciudadano imprescindible. 


\section{CONCLUSIONES}

A modo de conclusión podemos destacar la necesidad en una ciudad como Valencia, con un turismo urbano creciente, de actuaciones de diversificación turística que mejoren el flujo de visitantes en la zona y atraigan a nuevos segmentos de mercado promocionando los recursos patrimoniales. La propuesta descrita en este trabajo, entendida como primera aproximación a la idea del establecimiento de rutas turísticas industriales en el barrio de Patraix, da muestras del potencial existente en el barrio y las posibilidades que se abren de dar a conocer un patrimonio industrial poco conocido. Por su parte, el repaso y descripción del barrio de Patraix durante el primer tercio y la mitad del siglo XX ha sido posible gracias a la restitución completa que se ha hecho de la cartografía de 1929-44. El redibujo de esta cartografía, basándose en un trabajo anterior (Temes, 2007) complementado ahora con la correcta proyección cartográfica y la incorporación a un Sistema de Información Geográfica (SIG), abre la puerta a una potente herramienta de análisis que facilita la identificación del pasado industrial y su vinculación con el momento actual de la ciudad. El caso del pasado obrero industrial de Patraix puede entenderse como un buen ejemplo en el que la relectura de una cartografía intencionada nos ha ayudado a dar sentido a algunos de los escenarios urbanos hoy inconexos e incompletos de la ciudad de Valencia que pueden aportar nuevos valores para el turismo urbano.

\section{BIBLIOGRAFÍA}

AGUILAR CIVERA, I. (1998): Arquitectura industrial: concepto, método y fuentes. Valencia: Diputación de Valencia.

AGUILAR CIVERA, I. (2004): «El patrimonio arquitectónico industrial valenciano. Algunos ejemplos», Saitabi, Revista de la Facultat de Geografia i Història, $\mathrm{n}^{\circ}$ 54, pp. 155-192.

ALGARRA PARDO, V. (2003): «El urbanismo de Patraix en la huerta de la ciudad de Valencia (De la edad Media hasta 1940)», en Patraix: de pueblo a barrio. Valencia: Associació de Veïns i Cultural Patraix

BLAT PIZARRO, J. (2000): Vivienda obrera y crecimiento urbano: (Valencia 18531936). Valencia, Generalitat Valenciana, Conselleria d'Obres Públiques, Urbanisme i Transports.

CASANELLES RAHOLA, E. (1998): «Recuperación y uso del patrimonio industrial», Ábaco. Revista de Ciencias Sociales, n 19, pp. 11-18.

CASTELLS VALDIVIELSO, M. (2001): «Reencontrar el patrimonio. Estrategias de desarrollo territorial a partir de la interpretación», en $1{ }^{\circ}$ Congreso Virtual Internacional de Turismo Cultural. Disponible en: http://www.naya.org.ar/turismo/congreso/ inscriptos.htm.

CORBOZ, A. (1985): «Il territorio come palinsesto», Casabella, no 516, pp. 22-27.

CUCCIA T. y RIZZO I. (2011): «Tourism seasonality in cultural destinations: Empirical evidence from Sicily», Tourism Management, n³2(3), pp. 589-595.

DE CLUZEAU, C.O. (2000): Le Tourisme culturel. Paris, Presses Universitaires de France, $2^{\mathrm{a}}$ ed. 
FERNÁNDEZ, A.G., CERVANTES, M., MUÑIZ, N. y SANTOS, C. (2006): «Comparative Analysis of International Tourists in Inland Cultural Destinations: The Case of Castilla y León, Spain», en Cultural Tourism: Global and Local Perspectives, Haworth Press Inc, pp. 149-168.

FREEMAN, T. (1957): Interpreting our heritage. Carolina (EEUU). North Carolina, Press The University of North Carolina.

GIMÉNEZ BALDRÉS, E.J. (1996): Parcelaciones residenciales suburbanas: la formación de la periferia metropolitana de Valencia. Valencia, Editorial Universidad Politécnica de Valencia.

GREFFE, X. (2002): «Les rapports entre l'offre culturelle et le public touristique: une opportunité pour la culture, le tourisme et l'économie», en Cultura i turisme. Cultura y turismo. Culture et tourisme. Actas de Seminario, Girona, Universitat Girona, 55-74.

GUINOT I RODRÍGUEZ, E. (2007): «Una historia de la Huerta de Valencia», en El patrimonio hidráulico del Bajo Turia: L'Horta de València. vol.9. Valencia, Dirección General de Patrimonio.

LÓPEZ PATIÑO, MG. (2013). Chimeneas industriales de fábrica de ladrillo en el Levante y Sureste español. Influencia sobre otros territorios. Estudio y análisis de las tipologías constructivas. (Tesis doctoral). Editorial Universitat Politècnica de València. Disponible en: http://hdl.handle.net/10251/33181

LYNCH, K. (1998): La imagen de la ciudad. Barcelona, Gustavo Gili.

MALLOR, E., GONZÁLEZ-GALLARZA, M. y FAYOS, T. (2013): «¿Qué es y cómo se mide el Turismo Cultural? Un estudio longitudinal con series temporales para el caso Español», Pasos: Revista de turismo y patrimonio cultural, $\mathrm{n}^{\circ} 11(2)$, pp. 269-284.

MCKERCHER, B., CROS, H.D. y MCKERCHER, R.B. (2002): Cultural tourism: the partnership between tourism and cultural heritage management. Routledge Press.

PARADO ABAD, C. (2004): «La reutilización del patrimonio industrial como recurso turístico. Aproximación geográfica al turismo industrial», Treballs de la Societat Catalana de Geografía, $\mathrm{n}^{\circ}$ 57, pp. 7-32.

PIÉ R., VILANOVA J.M. y DÍAZ P. (2010): «Del Grand Tour al turismo de masas», Cuadernos del Territorio, $\mathrm{n}^{\circ} 2$, pp. 57-67.

SABATÉ BEL, J. (2004): «Paisajes culturales. El patrimonio como recurso básico para un nuevo modelo de desarrollo», Revista Urban, $\mathrm{n}^{\circ}$ 9, pp. 8-29.

SABATÉ BEL, J. (2011): «De la preservación del patrimonio a la ordenación del paisaje: intervenciones en paisajes culturales en Latinoamérica», en Agencia Española de Cooperación Internacional para el Desarrollo - AECID, $1^{\circ}$ Encuentro-Taller sobre Paisajes Culturales, Cartagena de Indias, pp. 11-23.

SABATÉ, J. y LISTA, A. (2001): «Casos d'estudi europeus», en Projectant l'eix del Llobregat. Paisatge cultural i desenvolupament regional, Barcelona, Universitat Politècnica de Catalunya-Massachusets Institute of Technology, pp. 78-90.

SAUER, C.O. (1925): The Morphology of Landscape. California, University of California Press. Publications in Geography.

SOLÀ-MORALES, M. (1981): «La identidad del territorio», Quaderns d'Arquitectura $i$ Urbanisme, Extra Comarcas, $\mathrm{n}^{\circ} 1(3)$. 
STYLIANOU-LAMBERT, T. (2010): «Gazing from home: Cultural tourism and art museums», Annals of Tourism Research, $\mathrm{n}^{\circ}$ 38(2), pp. 403-421.

TEMES CORDOVEZ, R. (2007): El tapiz de Penélope. Transformaciones residenciales sobre tejidos sin valor patrimonial (Tesis doctoral inédita). Departamento de Urbanismo. Valencia, Universidad Politécnica de Valencia. Disponible en: http://hdl.handle. net/10251/2906.

TEMES CORDOVEZ, R. (2008): «Las fuentes catastrales y la identificación de las transformaciones en los tejidos urbanos», CT: Catastro, n ${ }^{\circ}$ 4, pp. 55-78.

VALL, P. (1999): De colònies tèxtils a Parc Fluvial. El sistema de Colònies Tèxtils del Baix Berguedà. Gènesi i revaloraciò. Barcelona, Associaciò de Enginyers Industrials de Catalunya. 